NIST Special Publication 1200-13

\title{
Measurement of silver nanoparticle dissolution in complex biological and environmental matrices using UV/visible absorbance measurements
}

Version 1.0

J. Zook

R. MacCuspie

S. E. Long

E. J. Petersen

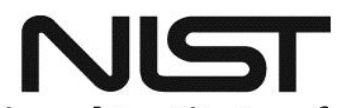

National Institute of Standards and Technology U.S. Department of Commerce 


\section{NIST Special Publication 1200-13 Measurement of silver nanoparticle dissolution in complex biological and environmental matrices using UV/visible absorbance measurements}

Version 1.0

J. Zook

E. J. Petersen

Biosystems and Biomaterials Division

Material Measurement Laboratory

R. I. MacCuspie

Florida Polytechnic University

Lakeland, FL

S. E. Long

Chemical Sciences Division

Material Measurement Laboratory

This publication is available free of charge from: http://dx.doi.org/10.6028/NIST.SP.13

May 2015

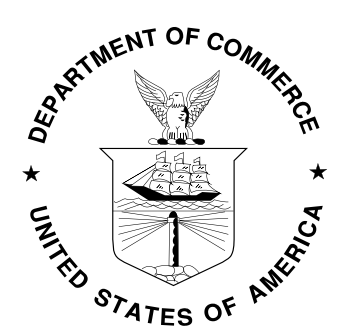

U.S. Department of Commerce Penny Pritzker, Secretary

National Institute of Standards and Technology Willie May, Acting Under Secretary of Commerce for Standards and Technology and Acting Director 
Certain commercial entities, equipment, or materials may be identified in this document in order to describe an experimental procedure or concept adequately. Such identification is not intended to imply recommendation or endorsement by the National Institute of Standards and Technology, nor is it intended to imply that the entities, materials, or equipment are necessarily the best available for the purpose.

National Institute of Standards and Technology Special Publication 1200-13 Natl. Inst. Stand. Technol. Spec. Publ. 1200-13, 10 pages (May 2015) CODEN: NSPUE2 This publication is available free of charge from: http://dx.doi.org/10.6028/NIST.SP.1200-13 


\section{FOREWORD}

This special publication is one in a series stemming from the National Nanotechnology Initiative (NNI) nanotechnology environmental health and safety (Nano-EHS) Research Strategy which identified Nanomaterial Measurement Infrastructure as one of the essential areas of research needed in order to develop an effective risk assessment and management plan regarding various aspects of nanotechnology in consumer products as it pertains to human health, exposure and the environment. The National Institute of Standards and Technology (NIST) was identified as a lead agency in the development of measurement strategies for the robust development to assess the potential effects of engineered nanomaterials and their fate in the environment. One important factor in these measurements is a reliable method to measure changes to engineered nanomaterials, such as particle dissolution, in biologically and environmentally relevant matrices.

The current protocol presents a method to measure dissolution of silver nanoparticles in biological and environmental matrices by measuring changes to the dispersions using ultravioletvisible (UV-vis) absorbance measurements. Updates to this protocol may be released in the future. Visit http://nist.gov/mml/np-measurement-protocols.cfm to check for revisions of this protocol, or new protocols in the series. We also encourage users to report citations to published work in which this protocol has been applied. 


\section{Introduction}

Making accurate measurements of the environmental fate and biological effects of engineered nanomaterials (ENMs) is critical for reliable risk assessment of these materials. However, results from toxicological studies, such as measurements of their cell toxicity, often differ substantially among laboratories and have questionable reliability (1-3). One important factor that can lead to variability in these measurements is that nanomaterials may undergo substantial changes such as agglomeration and dissolution in test media used to make toxicological measurements. If the changes to the nanomaterials are not accurately measured, this can lead to inaccurate dosing metrics (3). Silver nanoparticles (AgNPs), for example, can undergo numerous dynamic changes during nanoEHS studies such as agglomeration and dissolution (3-5). In addition, dissolved silver ions can form silver chloride nanoparticles in test media containing chloride $(3,4,6)$, and thus measurements of dissolved silver may not fully captured the extent of AgNP dissolution during an experiment. One challenge in measuring the changes to AgNPs in these systems is differentiating between $\mathrm{AgNPs}$ and $\mathrm{AgCl}$ particles. If measurements of total silver and dissolved silver are made by using techniques such as inductively-coupled plasma mass spectrometry before and after ultrafiltration, it is possible to differentiate between silver particles and dissolved silver. However, ultrafiltration may not distinguish between $\mathrm{AgNPs}$ and $\mathrm{AgCl}$ particles if they have similar sedimentation coefficients.

In this document, we describe a protocol, directly adapted from (4), to measure changes in the amount of silver remaining in the AgNP form for AgNPs with constant agglomeration states by quantifying the localized surface plasmon resonance (LSPR) using ultraviolet-visible (UV/vis) absorbance spectrum measurements. While AgNPs, for a given particle diameter, will give a consistent LSPR absorbance spectrum in the absence of agglomeration or dissolution (7), $\mathrm{AgCl}$ particles do not have an associated LSPR absorbance. $\mathrm{AgCl}$ particles may produce some light scattering at lower wavelengths, but this scattering can usually be subtracted since its spectrum is sufficiently different from the AgNP LSPR absorbance spectrum. Importantly, this technique also does not require the separation of the AgNPs from dissolved ions. When using the area under the absorbance peak from $340 \mathrm{~nm}$ to $650 \mathrm{~nm}$, the bias ( 2 x standard deviation) of the relationship between dissolution measured by inductively-coupled plasma-mass spectrometry (ICP-MS) and UV/vis spectroscopy with unagglomerated citrate-coated AgNPs that dissolved between $20 \%$ and $70 \%$ was $-0.5 \% \pm 5.4 \%$. The relationship between the integrated absorbance peak from $340 \mathrm{~nm}$ to $650 \mathrm{~nm}$ and the fractional dissolution from ICP-MS is shown in Figure A1.

\section{Principles and Scope}

This protocol is proposed for the measurement of AgNPs remaining in biological and environmental solutions using UV-vis absorbance spectroscopy as long as agglomeration of the AgNPs does not occur. Additional measurements are needed to quantify dissolved silver ions (i.e., ultracentrifugation), and this information along with those made in this protocol can provide information about the chemical speciation of silver in test media.

\section{Terminology}

This protocol complies with definitions relevant to nanotechnology as set forth in ASTM International E2456 (8) and is consistent with the draft standard ISO TS 80004-1:2010 (9). 
nanoparticle - sub-classification of ultrafine particle that is characterized by dimensions in the nanoscale (i.e., between approximately $1 \mathrm{~nm}$ and $100 \mathrm{~nm}$ ) in at least two dimensions; also referred to as "nano-object" in ISO TS 80004-1:2010 (9).

primary particle - the smallest discrete identifiable entity associated with a particle system; in this context, larger particle structures (e.g., aggregates and agglomerates) may be composed of primary particles.

aggregate - a discrete assemblage of primary particles strongly bonded together (i.e., fused, sintered, or metallically bonded).

Note-The adjective "primary", when used in conjunction with the term aggregate, is employed in the present context to indicate the smallest achievable dispersed particle entity.

agglomerate - assemblage of particles (including primary particles and/or smaller aggregates) held together by relatively weak forces (e.g., van der Waals, capillary, or electrostatic), that may break apart into smaller particles upon further processing.

Note-Although we define them as distinct entities, the terms aggregate and agglomerate have often been used interchangeably to denote particle assemblies.

dispersion - used in the present context to denote a liquid (aqueous) in which particles are homogeneously suspended, or the process of creating a suspension in which discrete particles are homogeneously distributed throughout a continuous fluid phase; implies the intention to break down agglomerates into their principal components (i.e., primary particles and/or aggregates).

\section{Materials and equipment}

\subsection{Reagents}

4.1.1 High-purity nitric acid

4.1.2 AgNPs dispersion in water (this protocol is for AgNPs already dispersed in water; the dispersion protocol for AgNPs in water and the synthesis of AgNPs are beyond the scope of this protocol)

4.1.3 Test media (e.g., de-ionized water, EPA moderately hard water with humic acid, or cell culture media Dulbecco's modified eagle medium (DMEM) with $4.5 \mathrm{~g} / \mathrm{L}$ glucose and sodium pyruvate but without phenol red or L-glutamine and with $2 \%$ bovine serum albumin added)

\subsection{Materials}

4.2.1 Centrifugation vials (e.g., Fisherbrand ${ }^{\mathrm{TM}}$ Snap-Cap ${ }^{\mathrm{TM}}$ Microcentrifuge Tubes: Flat Top $1.5 \mathrm{~mL}$ )

4.2.2 Autosampler plasticware (e.g. $15 \mathrm{~mL}$ round or conical polypropylene vials) for making ICP-MS measurements

4.2.3 Calibrated pipettes and disposable tips (e.g., Eppendorf ${ }^{\mathrm{TM}}$ Research $^{\mathrm{TM}}$ Plus Pipetters and Fisherbrand $^{\mathrm{TM}}$ Standard Pipette Tips)

4.2.4 1-cm quartz cuvette, or any cuvette that allows UV/vis measurements between $300 \mathrm{~nm}$ and $1100 \mathrm{~nm}$ 
4.2.5 Disposable semi-micro cuvettes for DLS measurements (e.g. Malvern 12mm Square Polystyrene Cuvettes (DTS0012))

\subsection{Equipment}

4.3.1 Inductively-coupled plasma mass spectrometer that is capable of making quantitative measurements of silver at concentrations at least one hundred times lower than the AgNP concentration to be tested

4.3.2 UV/vis absorbance spectrophotometer (single pass) capable of measuring between 300 $\mathrm{nm}$ and $1100 \mathrm{~nm}$

4.3.3 Dynamic light scattering instrument or any other instrument for quantifying the AgNP size distribution

4.3.4 Centrifuge capable of spinning the samples at a sufficiently fast concentration to separate the $\mathrm{AgNPs}$ and $\mathrm{AgCl}$ particles from ions (e.g., $20800 \mathrm{~g}$ for $30 \mathrm{~min}$ as described in 5.2.1)

\section{Measuring of changes in the AgNP concentration in complex media}

\subsection{UV/vis absorbance measurements}

5.1.1 Calibrate or validate the UV/vis spectrometer in the range $300 \mathrm{~nm}$ to $1100 \mathrm{~nm}$. This can be accomplished using, for example, NIST standard reference material 931g (liquid absorbance filters, UV-VIS) or 2031b (metal-on-fused-silica neutral density filters).

5.1.2 Measure the absorbance from $300 \mathrm{~nm}$ to $1100 \mathrm{~nm}$ of the test media in which AgNPs will be dispersed (without the AgNPs) relative to deionized water. The test media should be diluted with deionized water in the same way as the AgNP solutions in 5.1.3.

5.1.3 Measure the absorbance from $300 \mathrm{~nm}$ to $1100 \mathrm{~nm}$ of the initial AgNP samples. The AgNPs should be diluted with deionized water to approximately $5 \mathrm{ug} / \mathrm{mL}$ or whatever concentration results in peak absorbance values between 0.5 and 1.0. All AgNP samples should be vortexed immediately prior to measurement here and in 5.1.4 in order to avoid artifacts from sedimentation of agglomerates.

5.1.4 Measure the absorbance from $300 \mathrm{~nm}$ to $1100 \mathrm{~nm}$ of the AgNP samples in the test media at different time points. It is important to also measure AgNP agglomeration (e.g., using dynamic light scattering as described in (10)) as agglomeration will impact the absorbance peak and could result in an incorrect estimation of the AgNP dissolution.

5.1.5 Subtract the absorbance of the media from that of the AgNP samples.

5.1.6 To minimize effects of slight changes in the baseline, the baseline (mean absorbance between $900 \mathrm{~nm}$ and 1,100 nm) can be subtracted from each curve (4). For samples with $\mathrm{Cl}$ present (this will be known for test media commercially purchased or prepared in the laboratory), subtract the absorbance of $\mathrm{AgCl} \mathrm{NPs}$ from the spectrum using the equation $\mathrm{Abs}_{\mathrm{AgCl}}=\left(\mathrm{Abs}_{325}-0.055 \mathrm{xAbs}_{400}\right) \times(\lambda / 325)^{-4}$. To develop this equation (4), scattering due to $\mathrm{AgCl}$ NPs was approximated by assuming Rayleigh scattering (absorbance is proportional to $\left.\lambda^{-4}\right)$ and setting the absorbance at $325 \mathrm{~nm}\left(\mathrm{Abs}_{325}\right)$ to $5.5 \%$ of the peak absorbance $\left(\mathrm{Abs}_{400}\right)$; unagglomerated AgNPs are known to have an LSPR near $400 \mathrm{~nm}$. For example, the absorbance reported at $400 \mathrm{~nm}$ by the spectrophotometer may be the absorbance between 399.5 and 400.5 if it reports values at $1 \mathrm{~nm}$ increments. Note that different sizes of AgNPs may have peak absorbances that differ from $400 \mathrm{~nm}$, and in this case the actual peak absorbance value should be used. The absorbance at $325 \mathrm{~nm}$ was 
chosen for fitting because unagglomerated AgNPs have a minimum at $325 \mathrm{~nm}$, and 5.5\% was used because $\mathrm{Abs}_{325}$ is 5.5\% of $\mathrm{Abs}_{400}$ for unagglomerated AgNPs. This correction factor will be most accurate when $\mathrm{AgCl}$ NPs are small, because deviations from Rayleigh scattering become significant when the NPs are not much smaller than the wavelength of light.

5.1.7 Integrate the peak from $340 \mathrm{~nm}$ to $650 \mathrm{~nm}$. This can be accomplished by adding the absorbance values between $340 \mathrm{~nm}$ and $650 \mathrm{~nm}$ using software such as Microsoft Excel. For example, if the spectrometer measures absorbance at increments of $1 \mathrm{~nm}$, add all 311 absorbance measurements between $340 \mathrm{~nm}$ and $650 \mathrm{~nm}$. The percentage decrease in the integrated peak area will give you an estimate of the AgNP dissolution for a sample without agglomeration at whatever time points are measured as described in 5.1.4.

\subsection{Measuring AgCl NP formation}

5.2.1 Measure the formation of silver ions in the AgNP samples (e.g., centrifugation at a high speed (20800 g for $30 \mathrm{~min}$ ) followed by elemental analysis of the supernatant (e.g., using ICP-MS as described in (4))). The accuracy of different measurement methods for quantifying silver ion dissolution is beyond the scope of this protocol.

5.2.2 Subtract the amount of total AgNP dissolution (determined in step 5.1.7) from the total mass of dissolved silver (determined in step 5.2.1); to convert from the dissolved silver concentration to the total mass of dissolved silver, multiply the dissolved silver concentration by the volume of solution.

\section{Abbreviations}

$\begin{array}{ll}\text { AgNP } & \text { silver nanoparticle } \\ \text { AgCl } & \text { silver chloride } \\ \text { DLS } & \text { dynamic light scattering } \\ \text { DMEM } & \text { Dulbecco's modified eagle medium } \\ \text { ENM } & \text { engineered nanomaterial } \\ \text { EPA } & \text { environmental protection agency } \\ \text { ICP-MS } & \text { inductively-coupled plasma-mass spectrometry } \\ \text { LSPR } & \text { localized surface plasmon resonance } \\ \text { NP } & \text { nanoparticle } \\ \text { UV/vis } & \text { ultraviolet-visible }\end{array}$

\section{Acknowledgements}

We acknowledge the contributions of Danielle Cleveland and Carly Lay A. Geronimo who contributed to manuscript (4) from which this protocol was adapted.

\section{$8 \underline{\text { References }}$}

1. Krug, H. F.; Wick, P. Angewandte Chemie (International ed in English) 2011, 50 (6), 1260-1278.

2. $\quad$ Schrurs, F.; Lison, D. Nat Nano 2012, 7 (9), 546-548.

3. Petersen, E. J.; Henry, T. B.; Zhao, J.; MacCuspie, R. I.; Kirschling, T. L.; Dobrovolskaia, M. A.; Hackley, V.; Xing, B.; White, J. C. Environ. Sci. Technol. 2014, 48 (8), 4226-4246. 
4. Zook, J. M.; Long, S. E.; Cleveland, D.; Geronimo, C. L. A.; MacCuspie, R. I. Anal. Bioanal. Chem. 2011, 401 (6), 1993-2002.

5. Zook, J. M.; MacCuspie, R. I.; Locascio, L. E.; Halter, M. D.; Elliott, J. T. Nanotoxicology 2011, 5 (4), 517-530.

6. Lowry, G. V.; Gregory, K. B.; Apte, S. C.; Lead, J. R. Environ. Sci. Technol. 2012, 46 (13), 68936899.

7. Gorham, J. M.; MacCuspie, R. I.; Klein, K. L.; Fairbrother, D. H.; Holbrook, R. D. J. Nano. Res. 2012, 14 (10), 1-16.

8. $\quad$ ASTM (American Society for Testing Materials) International, E2456-06: standard terminology relating to nanotechnology. West Conshohocken, PA., 2006.

9. ISO (International Organization for Standardization), TS 80004-1: nanotechnologies vocabulary - Part 1: Core terms. Geneva, Switzerland, 2010.

10. Hackley, V. A.; Clogston, J. D., NIST-NCL joint assay protocol PCC-1: measuring the size of nanoparticles in aqueous media using batch-mode dynamic light scattering. Available at http://ncl.cancer.gov/working assay-cascade.asp, 2007. 
Appendix

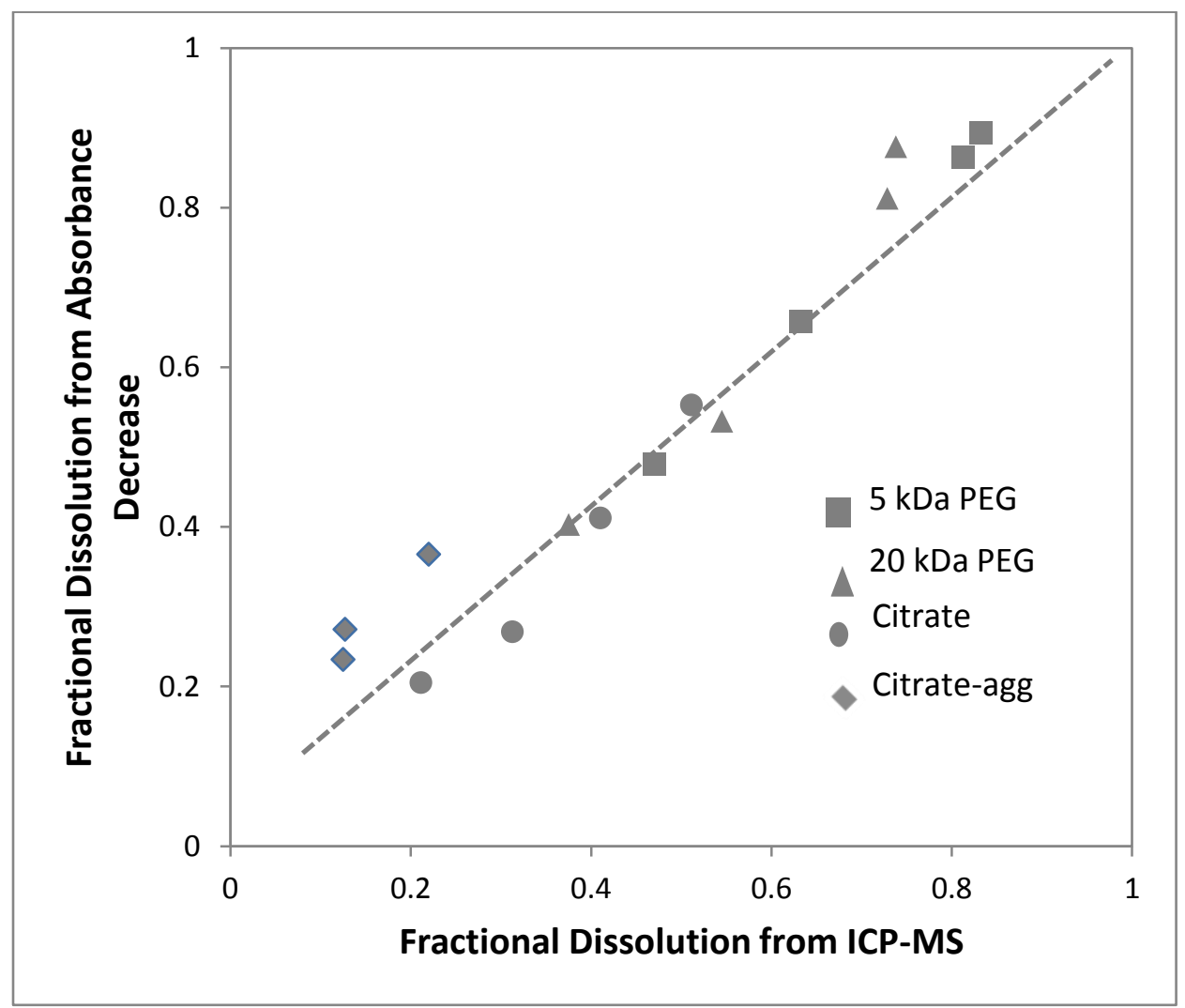

Figure A1 Comparison of fractional dissolution of AgNPs measured by decrease in absorbance to dissolution measured by ICP-MS. The decrease in absorbance was calculated from the integrated area under the absorbance curve. Dissolution was measured for AgNPs coated with (1) 5 kDa PEG-thiol (squares) and (2) $20 \mathrm{kDa}$ PEG-thiol (triangles) in $100 \mathrm{mmol} / \mathrm{L} \mathrm{HNO3,} \mathrm{(3)}$ citrate-coated AgNPs dispersed as primary particles in $150 \mathrm{mmol} / \mathrm{L} \mathrm{KNO} 3+2 \% \mathrm{BSA}$ (circles), and (4) citrate-coated AgNPs dispersed as agglomerates that grow over 5 days from $230 \mathrm{~nm}$ to $780 \mathrm{~nm}$ (DLS intensity-weighted mean diameters) in $7.5 \mathrm{mmol} / \mathrm{L} \mathrm{KNO3+0.1 \%} \mathrm{BSA} \mathrm{(diamonds).}$ The diagonal dotted grey line indicates the 1:1 correlation expected from Beer's law. Standard deviations for replicated absorbance and ICP-MS measurements of the same sample are smaller than the symbols. 